\title{
EXISTENCE OF ENTROPY SOLUTIONS FOR SOME NONLINEAR PROBLEMS IN ORLICZ SPACES
}

\section{A. BENKIRANE AND J. BENNOUNA}

Received 20 October 2001

We study in the framework of Orlicz Sobolev spaces $W_{0}^{1} L_{M}(\Omega)$, the existence of entropic solutions to the nonlinear elliptic problems: $-\operatorname{div} a(x, u, \nabla u)+\operatorname{div} \phi(u)$ $=f$ in $\Omega$, for the case where the second member of the equation $f \in L^{1}(\Omega)$, and $\phi \in\left(C^{0}(\mathbb{R})\right)^{N}$.

\section{Introduction}

Let $\Omega$ be a bounded open subset of $\mathbb{R}^{N}$ and let $A(u)=-\operatorname{div} a(x, u, \nabla u)$ be a Leray-Lions operator defined on $W_{0}^{1, p}(\Omega), 1<p<\infty$.

We consider the nonlinear elliptic problem

$$
\begin{aligned}
-\operatorname{div} a(x, u, \nabla u) & =f-\operatorname{div} \phi(u) & & \text { in } \Omega, \\
u & =0 & & \text { on } \partial \Omega,
\end{aligned}
$$

where

$$
f \in L^{1}(\Omega), \quad \phi \in\left(C^{0}(\mathbb{R})\right)^{N} .
$$

Note that no growth hypothesis is assumed on the function $\phi$, which implies that the term $\operatorname{div} \phi(u)$ may be meaningless, even as a distribution. The notion of entropy solution, used in [8], allows us to give a meaning to a possible solution of (1.1).

In fact Boccardo proved in [8], for $p$ such that $2-1 / N<p<N$, the existence and regularity of an entropy solution $u$ of problem (1.1), that is,

$$
\begin{array}{ll}
u \in W_{0}^{1, q}(\Omega), & q<\tilde{p}=\frac{(p-1) N}{N-1}, \\
T_{k}(u) \in W_{0}^{1, p}(\Omega), & \forall k>0,
\end{array}
$$




$$
\begin{gathered}
\int_{\Omega} a(x, u, \nabla u) \nabla T_{k}[u-\varphi] d x \leq \int_{\Omega} f T_{k}[u-\varphi] d x+\int_{\Omega} \phi(u) \nabla T_{k}[u-\varphi] d x \\
\forall \varphi \in W_{0}^{1, p}(\Omega) \cap L^{\infty}(\Omega),
\end{gathered}
$$

where

$$
T_{k}(s)=s \quad \text { if }|s| \leq k \quad T_{k}(s)=k \frac{s}{|s|} \quad \text { if }|s|>k
$$

For the case $\phi=0$ and $f$ is a bounded measure, Bénilan et al. proved in [3] the existence and uniqueness of entropy solutions.

We mention as a parallel development, the work of Lions and Murat [14] who consider similar problems in the context of the renormalized solutions introduced by Diperna and Lions [10] for the study of the Boltzmann equations. They can prove existence and uniqueness of renormalized solution.

The functional setting in these works is that of the usual Sobolev space $W^{1, p}$. Accordingly, the function $a$ is supposed to satisfy polynomial growth conditions with respect to $u$ and its derivatives $\nabla u$. When trying to generalize the growth condition on $a$, one is led to replace $W^{1, p}$ by a Sobolev space $W^{1} L_{M}$ built from an Orlicz space $L_{M}$ instead of $L^{p}$. Here the $N$-function $M$ which defines $L_{M}$ is related to the actual growth of the function $a$.

It is our purpose, in this paper, to prove the existence of entropy solution for problem (1.1) in the setting of the Orlicz Sobolev space $W_{0}^{1} L_{M}(\Omega)$. Our result, Theorem 3.5, generalizes [8, Theorem 2.1] and gives in particular a refinement of his result (see Remark 3.6).

For some existence results for strongly nonlinear elliptic equations in Orlicz spaces $[4,5,6]$.

\section{Preliminaries}

2.1. Let $M: \mathbb{R}^{+} \rightarrow \mathbb{R}^{+}$be an $N$-function, that is, $M$ is continuous, convex, with $M(t)>0$ for $t>0, M(t) / t \rightarrow 0$ as $t \rightarrow 0$ and $M(t) / t \rightarrow \infty$ as $t \rightarrow \infty$.

Equivalently, $M$ admits the representation $M(t)=\int_{0}^{t} a(\tau) d \tau$, where $a$ : $\mathbb{R}^{+} \rightarrow \mathbb{R}^{+}$is nondecreasing, right continuous, with $a(0)=0, a(t)>0$ for $t>0$ and $a(t) \rightarrow \infty$ as $t \rightarrow \infty$.

In the following, we assume, for convenience, that all $N$-functions are twice continuously differentiable, see Benkirane and Gossez [7].

The $N$-function $\bar{M}$ conjugate to $M$ is defined by $\bar{M}(t)=\int_{0}^{t} \bar{a}(\tau) d \tau$, where $\bar{a}: \mathbb{R}^{+} \rightarrow \mathbb{R}^{+}$is given by $\bar{a}(t)=\sup \{s: a(s) \leq t\}$, see $[1,13]$.

The $N$-function $M$ is said to satisfy the $\Delta_{2}$-condition (resp., near infinity) if for some $k$ and for every $t \geq 0$,

$$
\left.M(2 t) \leq k M(t) \quad \text { (resp., for } t \geq \text { some } t_{0}\right) .
$$


Let $M$ and $P$ be two $N$-functions. The notation $P \ll M$ means that $P$ grows essentially less rapidly than $M$, that is, for each $\epsilon>0, P(t) / M(\epsilon t) \rightarrow 0$ as $t \rightarrow \infty$. This is the case if and only if $\lim _{t \rightarrow \infty} M^{-1}(t) / P^{-1}(t)=0$. We will extend all $N$ functions into even functions on all $\mathbb{R}$.

2.2. Let $\Omega$ be an open subset of $\mathbb{R}^{N}$. The Orlicz class $K_{M}(\Omega)$ (resp., the Orlicz space $L_{M}(\Omega)$ ) is defined as the set of (equivalence classes of) real-valued measurable functions $u$ on $\Omega$ such that

$$
\int_{\Omega} M(u(x)) d x<\infty
$$

(resp., $\int_{\Omega} M(u(x) / \lambda) d x<\infty$ for some $\lambda>0$ ). The space $L_{M}(\Omega)$ is a Banach space under the norm

$$
\|u\|_{M}=\inf \left\{\lambda>0: \int_{\Omega} M\left(\frac{u(x)}{\lambda}\right) d x \leq 1\right\}
$$

and $K_{M}(\Omega)$ is a convex subset of $L_{M}(\Omega)$. The closure in $L_{M}(\Omega)$ of the set of bounded measurable functions with compact support in $\bar{\Omega}$ is denoted by $E_{M}(\Omega)$.

The equality $E_{M}(\Omega)=L_{M}(\Omega)$ holds if and only if $M$ satisfies the $\Delta_{2}$ condition, for all $t$ or for $t$ large according to whether $\Omega$ has infinity measure or not.

The dual of $E_{M}(\Omega)$ can be identified with $L_{\bar{M}}(\Omega)$ by means of the pairing $\int_{\Omega} u(x) v(x) d x$, and the dual norm on $L_{\bar{M}}(\Omega)$ is equivalent to $\|\cdot\|_{\bar{M}}$. We say that $u_{n}$ converges to $u$ for the modular convergence in $L_{M}(\Omega)$ if for some $\lambda>0$

$$
\int_{\Omega} M\left(\frac{\left|u_{n}-u\right|}{\lambda}\right) d x \rightarrow 0 \quad \text { as } n \longrightarrow \infty
$$

If $M$ satisfies the $\Delta_{2}$-condition, then the modular convergence coincide with the norm convergence.

2.3. The Orlicz Sobolev space $W^{1} L_{M}(\Omega)$ (resp., $W^{1} E_{M}(\Omega)$ ) is the space of all functions $u$ such that $u$ and its distributional derivatives up to order one lie in $L_{M}(\Omega)$ (resp., $E_{M}(\Omega)$ ). It is a Banach space under the norm

$$
\|u\|_{1, M}=\sum_{|\alpha| \leq 1}\left\|D^{\alpha} u\right\|_{M}
$$

Thus, $W^{1} L_{M}(\Omega)$ and $W^{1} E_{M}(\Omega)$ can be identified with subspaces of the product of $N+1$ copies of $L_{M}(\Omega)$. Denoting this product by $\Pi L_{M}$, we will use the weak topologies $\sigma\left(\prod L_{M}, \prod E_{\bar{M}}\right)$ and $\sigma\left(\prod L_{M}, \prod L_{\bar{M}}\right)$.

The space $W_{0}^{1} E_{M}(\Omega)$ is defined as the norm closure of $\mathscr{D}(\Omega)$ in $W^{1} E_{M}(\Omega)$ and the space $W_{0}^{1} L_{M}(\Omega)$ as the $\sigma\left(\prod L_{M}, \prod E_{\bar{M}}\right)$ closure of $\mathscr{D}(\Omega)$ in $W^{1} L_{M}(\Omega)$. 
We say that $u_{n}$ converges to $u$ for the modular convergence in $W^{1} L_{M}(\Omega)$ if for some $\lambda>0$

$$
\int_{\Omega} M\left(\frac{\left|D^{\alpha} u_{n}-D^{\alpha} u\right|}{\lambda}\right) d x \rightarrow 0 \quad \forall|\alpha| \leq 1
$$

This implies the convergence $\sigma\left(\prod L_{M}, \prod L_{\bar{M}}\right)$.

2.4. Let $W^{-1} L_{\bar{M}}(\Omega)$ (resp., $W^{-1} E_{\bar{M}}(\Omega)$ ) denote the space of distributions on $\Omega$ which can be written as sums of derivatives of order $\leq 1$ of functions in $L_{\bar{M}}(\Omega)$ (resp., $E_{\bar{M}}(\Omega)$ ). It is a Banach space under the usual quotient norm.

If the open set $\Omega$ has the segment property, then the space $\mathscr{D}(\Omega)$ is dense in $W_{0}^{1} L_{M}(\Omega)$ for the modular convergence and thus for the topology $\sigma\left(\prod L_{M}\right.$, $\left.\prod L_{\bar{M}}\right)$. Consequently, the action of a distribution in $W^{-1} L_{\bar{M}}(\Omega)$ on an element of $W_{0}^{1} L_{M}(\Omega)$ is well defined.

2.5. We recall the following lemmas.

Lemma 2.1 (see [5]). Let $\Omega$ be an open subset of $\mathbb{R}^{N}$ with finite measure. Let $M, P$, and $Q$ be $N$-functions such that $Q \ll P$, and let $f: \Omega \times \mathbb{R} \rightarrow \mathbb{R}^{N}$ be a Carathéodory function such that

$$
|f(x, s)| \leq c(x)+k_{1} P^{-1} M\left(k_{2}|s|\right) \quad \text { a.e. } x \in \Omega, \forall s \in \mathbb{R}
$$

where $k_{1}, k_{2} \in \mathbb{R}_{+}, c(x) \in E_{Q}(\Omega)$. Let $N_{f}$ be the Nemytskii operator defined from $P\left(E_{M}(\Omega), 1 / k_{2}\right)=\left\{u \in L_{M}(\Omega): d\left(u, E_{M}(\Omega)\right)<1 / k_{2}\right\}$ to $\left(E_{Q}(\Omega)\right)^{N}$ by $N_{f}(u)(x)=$ $f(x, u(x))$. Then $N_{f}$ is strongly continuous.

Lemma 2.2 (see [5]). Let $F: \mathbb{R} \rightarrow \mathbb{R}$ be uniformly Lipschitzian, with $F(0)=0$. Let $M$ be an $N$-function and let $u \in W_{0}^{1} L_{M}(\Omega)$ (resp., $W_{0}^{1} E_{M}(\Omega)$ ). Then $F(u) \in$ $W_{0}^{1} L_{M}(\Omega)$ (resp., $W_{0}^{1} E_{M}(\Omega)$ ). Moreover, if the set $D$ of discontinuity points of $F^{\prime}$ is finite, then

$$
\frac{\partial}{\partial x_{i}} F(u)= \begin{cases}F^{\prime}(u) \frac{\partial u}{\partial x_{i}} & \text { a.e. in }\{x \in \Omega: u(x) \notin D\} \\ 0 & \text { a.e. in }\{x \in \Omega: u(x) \in D\} .\end{cases}
$$

Then $F: W_{0}^{1} L_{M}(\Omega) \rightarrow W_{0}^{1} L_{M}(\Omega)$ is sequentially continuous with respect to the weak $^{*}$ topology $\sigma\left(\prod L_{M}, \prod E_{\bar{M}}\right)$.

Lemma 2.3 (see [11]). Let $\Omega$ have the segment property. Then for each $v \in$ $W_{0}^{1} L_{M}(\Omega)$, there exists a sequence $v_{n} \in \mathscr{D}(\Omega)$ such that $v_{n}$ converges to $v$ for the modular convergence in $W_{0}^{1} L_{M}(\Omega)$. Furthermore, if $v \in W_{0}^{1} L_{M}(\Omega) \cap L^{\infty}(\Omega)$ then

$$
\left\|v_{n}\right\|_{L^{\infty}(\Omega)} \leq(N+1)\|v\|_{L^{\infty}(\Omega)} .
$$


2.6. We introduce the following notation, see $[2,15]$.

Definition 2.4. Let $M$ be an $N$-function, and define the following set:

$$
\begin{aligned}
\mathscr{A}_{M}=\{ & Q: Q \text { is an } N \text {-function such that } \frac{Q^{\prime \prime}}{Q^{\prime}} \leq \frac{M^{\prime \prime}}{M^{\prime}}, \\
& \left.\int_{0}^{1} Q \circ H^{-1}\left(\frac{1}{r^{1-1 / N}}\right) d r<\infty \text { where } H(r)=\frac{M(r)}{r}\right\} .
\end{aligned}
$$

Remark 2.5. Let $M(t)=t^{p}$ and $Q(t)=t^{q}$, then the condition $Q \in \mathscr{A}_{M}$ is equivalent to the following conditions:

(i) $2-1 / N<p<N$

(ii) $q<\tilde{p}=(p-1) N /(N-1)$, see (1).

Remark 2.6. We can give some examples of $N$-functions $M$ for which the set $\mathscr{A}_{M}$ is not empty. Here, the $N$-functions $M$ are defined only at infinity.

(1) For $M(t)=t^{2} \log t$ and $Q(t)=t \log t$, we have $H(t)=t \log t$ and $H^{-1}(t)=$ $t(\log t)^{-1}$ at infinity, see [13]. Then the $N$-function $Q$ belongs to $\mathscr{A}_{M}$.

(2) For $M(t)=t^{2} \log ^{2} t$ at infinity and $Q(t)=t \log ^{2} t$, we have $H(t)=t \log ^{2} t$ and $H^{-1}(t)=t(\log t)^{-2}$ at infinity, see [13]. Then the $N$-function $Q$ belongs to $\mathscr{A}_{M}$.

\section{Definition and existence of entropy solutions}

Let $\Omega$ be a bounded open subset of $\mathbb{R}^{N}$ with the segment property. Let $M, P$ be two $N$-functions such that $P \ll M$.

Let $A: D(A) \subset W_{0}^{1} L_{M}(\Omega) \rightarrow W^{-1} L_{\bar{M}}(\Omega)$ be a mapping (not defined everywhere) given by $A(u)=-\operatorname{div} a(x, u, \nabla u)$ where $a: \Omega \times \mathbb{R} \times \mathbb{R}^{N} \rightarrow \mathbb{R}^{N}$ is a Carathéodory function satisfying for a.e. $x \in \Omega$ and all $t \in \mathbb{R}, \xi$, $\bar{\xi}$ with $\xi \neq \bar{\xi}$,

$$
\begin{gathered}
|a(x, t, \xi)| \leq d(x)+k_{1} \bar{P}^{-1} M\left(k_{2}|t|\right)+k_{3} \bar{M}^{-1} M\left(k_{4}|\xi|\right), \\
{[a(x, t, \xi)-a(x, t, \bar{\xi})][\xi-\bar{\xi}]>0,} \\
a(x, t, \xi) \xi \geq \alpha M\left(\frac{|\xi|}{\lambda}\right)
\end{gathered}
$$

where $d(x) \in E_{\bar{M}}(\Omega), d \geq 0, \alpha, \lambda \in \mathbb{R}_{+}^{*}, k_{1}, k_{2}, k_{3}, k_{4} \in \mathbb{R}_{+}$.

Consider the nonlinear elliptic problem (1.1) where

$$
f \in L^{1}(\Omega)
$$

and $\phi=\left(\phi_{1}, \ldots, \phi_{N}\right)$ satisfies

$$
\phi \in\left(C^{0}(\mathbb{R})\right)^{N} .
$$

As in [8], we define the following notion of an entropy solution, which gives a meaning to a possible solution of (1.1). 
Definition 3.1. Assume that (3.1), (3.2), (3.3), (3.4), and (3.5) hold true and $\mathscr{A}_{M} \neq \emptyset$. A function $u$ is an entropy solution of problem (1.1) if

$$
\begin{gathered}
u \in W_{0}^{1} L_{Q}(\Omega) \quad \forall Q \in \mathscr{A}_{M}, \\
T_{k}(u) \in W_{0}^{1} L_{M}(\Omega) \quad \forall k>0, \\
\int_{\Omega} a(x, u, \nabla u) \nabla T_{k}[u-\varphi] d x \leq \int_{\Omega} f T_{k}[u-\varphi] d x+\int_{\Omega} \phi(u) \nabla T_{k}[u-\varphi] d x \\
\forall \varphi \in W_{0}^{1} L_{M}(\Omega) \cap L^{\infty}(\Omega) .
\end{gathered}
$$

We cannot use the solution $u$ as a test function in (1.1), because $u$ does not belong to $W_{0}^{1} L_{M}(\Omega)$. An important role is played by $T_{k}(u)$ and the test functions

$$
T_{k}[u-\varphi], \quad \varphi \in W_{0}^{1} L_{M}(\Omega) \cap L^{\infty}(\Omega)
$$

because both belong to $W_{0}^{1} L_{M}(\Omega)$.

In Theorem 3.5, we prove the existence of solution of problem (1.1), in the framework of entropy solutions.

LEMMA 3.2. Let $\Omega$ be a bounded open subset of $\mathbb{R}^{N}$ with the segment property. If $u \in\left(W_{0}^{1} L_{M}(\Omega)\right)^{N}$ then $\int_{\Omega} \operatorname{div} u d x=0$.

Proof of Lemma 3.2. It is sufficient to use an approximation of $u$.

We recall the following lemma (see [15, Lemma 2]).

Lemma 3.3. Let $M$ be an $N$-function, $u \in W^{1} L_{M}(\Omega)$ such that $\int_{\Omega} M(|\nabla u|) d x<\infty$, then

$$
\begin{aligned}
-\mu^{\prime}(t) \geq & N C_{N}^{1 / N} \mu^{1-1 / N}(t) \\
& \times C\left(\frac{-1}{N C_{N}^{1 / N} \mu^{1-1 / N}(t)} \frac{d}{d t} \int_{\{|u|>t\}} M(|\nabla u|) d x\right) \quad \forall t>0,
\end{aligned}
$$

where $\mathrm{C}$ is the function defined as

$$
\mathrm{C}(t)=\frac{1}{\sup \{r \geq 0, H(r) \leq t\}}, \quad H(r)=\frac{M(r)}{r} .
$$

The function $C_{N}$ is the measure of the unit ball of $\mathbb{R}^{N}$, and $\mu(t)=$ meas $\{|u|>t\}$.

Lemma 3.4. Let $(X, \tau, \mu)$ be a measurable set such that $\mu(X)<\infty$. Let $\gamma$ be a measurable function $\gamma: X \rightarrow[0, \infty)$ such that

$$
\mu(\{x \in X: \gamma(x)=0\})=0,
$$

then for each $\epsilon>0$, there exists $\delta>0$ such that $\int_{A} \gamma(x) d x<\delta$ implies

$$
\mu(A) \leq \epsilon .
$$


Theorem 3.5. Under assumptions (3.1), (3.2), (3.3), (3.4), and (3.5), with $\mathscr{A}_{M} \neq$ $\emptyset$, there exists an entropy solution $u$ of problem (1.1) (in the sense of Definition 3.1).

Remark 3.6. In the case $M(t)=t^{p}$, Theorem 3.5 gives a refinement of the regularity result (1) (i.e., $\left.u \in W_{0}^{1, q}(\Omega), q<\tilde{p}=((p-1) N / N-1)\right)$. In fact, by Theorem 3.5, we have $u \in W_{0}^{1} L_{Q}(\Omega)$ for each $Q \in \mathscr{A}_{M}$ (for example for $Q(t)=t \tilde{p} / \log ^{\alpha}(e+$ $t), \alpha>1)$.

Proof of Theorem 3.5

Step 1. Define, for each $n>0$, the approximations

$$
\phi_{n}(s)=\phi\left(T_{n}(s)\right), \quad f_{n}(s)=T_{n}[f(s)] .
$$

Consider the nonlinear elliptic problem

$$
u_{n} \in W_{0}^{1} L_{M}(\Omega), \quad-\operatorname{div} a\left(x, u_{n}, \nabla u_{n}\right)=f_{n}-\operatorname{div} \phi_{n}\left(u_{n}\right) \quad \text { in } \Omega .
$$

From Gossez and Mustonen [12, Proposition 1, Remark 2], problem (3.13) has at least one solution.

Step 2. We will prove that $\left(u_{n}\right)$ is bounded in $W_{0}^{1} L_{Q}(\Omega)$ for each $Q \in \mathscr{A}_{M}$. Let $\varphi$ be the truncation defined, for each $t, h>0$, by

$$
\varphi(\xi)= \begin{cases}0 & \text { if } 0 \leq \xi \leq t, \\ \frac{1}{h}(\xi-t) & \text { if } t<\xi<t+h \\ 1 & \text { if } \xi \geq t+h \\ -\varphi(-\xi) & \text { if } \xi<0\end{cases}
$$

Using the test function $v=\varphi\left(u_{n}\right)$ in (3.13) $\left(v \in W_{0}^{1} L_{M}(\Omega)\right.$ by Lemma 2.2), we have

$$
\int_{\Omega} a\left(x, u_{n}, \nabla u_{n}\right) \varphi^{\prime}\left(u_{n}\right) \nabla u_{n} d x=\int_{\Omega} f_{n} \varphi\left(u_{n}\right) d x+\int_{\Omega} \phi_{n}\left(u_{n}\right) \nabla \varphi\left(u_{n}\right) d x .
$$

We claim now that

$$
\int_{\Omega} \phi_{n}\left(u_{n}\right) \nabla \varphi\left(u_{n}\right) d x=0
$$

Indeed,

$$
\nabla \varphi\left(u_{n}\right)=\varphi^{\prime}\left(u_{n}\right) \nabla u_{n},
$$

where

$$
\varphi^{\prime}(\xi)= \begin{cases}\frac{1}{h} & \text { if } t<|\xi|<t+h \\ 0 & \text { otherwise }\end{cases}
$$


92 Entropy solutions in Orlicz spaces

define $\theta(s)=\phi_{n}(s)(1 / h) \chi_{\{t<|s|<t+h\}}$, and $\tilde{\theta}(s)=\int_{0}^{s} \theta(\tau) d \tau$, we have by Lemma 2.2, $\tilde{\theta}\left(u_{n}\right) \in\left(W_{0}^{1} L_{M}(\Omega)\right)^{N}$, which implies

$$
\begin{aligned}
\int_{\Omega} \phi_{n}\left(u_{n}\right) \nabla \varphi\left(u_{n}\right) d x & =\int_{\Omega} \phi_{n}\left(u_{n}\right) \frac{1}{h} \chi_{\left\{t<\left|u_{n}\right|<t+h\right\}} \nabla u_{n} d x=\int_{\Omega} \theta\left(u_{n}\right) \nabla u_{n} d x \\
& =\int_{\Omega} \operatorname{div}\left(\tilde{\theta}\left(u_{n}\right)\right) d x=0 \quad \text { (see Lemma 3.2). }
\end{aligned}
$$

This proves (3.16). By (3.3) and (3.15), we have (where we can suppose without loss of generality that $\lambda=1$, since one can take $\left.u_{n}^{\prime}=u_{n} / \lambda\right)$

$$
\frac{\alpha}{h} \int_{t<\left|u_{n}\right|<t+h} M\left(\left|\nabla u_{n}\right|\right) d x \leq\|f\|_{1, \Omega} .
$$

Let $h \rightarrow 0$, then

$$
-\frac{d}{d t} \int_{\left\{\left|u_{n}\right|>t\right\}} M\left(\left|\nabla u_{n}\right|\right) d x \leq C \quad \text { with } C=\frac{\|f\|_{1, \Omega}}{\alpha} .
$$

We prove the following inequality, which allows us to obtain the boundedness of $\left(u_{n}\right)$ in $W_{0}^{1} L_{Q}(\Omega)$,

$$
\begin{aligned}
& -\frac{d}{d t} \int_{\left|u_{n}\right|>t} Q\left(\left|\nabla u_{n}\right| d x\right) \\
& \quad \leq-\mu_{n}^{\prime}(t) Q \circ H^{-1}\left(-\frac{1}{N C_{N}^{1 / N} \mu_{n}(t)^{1-1 / N}} \frac{d}{d t} \int_{\left\{\left|u_{n}\right|>t\right\}} M\left(\left|\nabla u_{n}\right|\right) d x\right) .
\end{aligned}
$$

Indeed, let $\mathrm{C}(s)=1 / H^{-1}(s)$, where $H(r)=M(r) / r$ and $H^{-1}(s)=\sup \{r \geq 0$, $H(r) \leq s\}$. Then

$$
\mathrm{C}(s)=\frac{s}{M \circ H^{-1}(s)} .
$$

By Lemma 3.3 we have, with $\mu_{n}(t)=$ meas $\left\{\left|u_{n}\right|>t\right\}$,

$$
\begin{aligned}
-\mu_{n}^{\prime}(t) \geq & N C_{N}^{1 / N} \mu_{n}(t)^{1-1 / N} \\
& \times C\left(-\frac{1}{N C_{N}^{1 / N}} \mu_{n}(t)^{1-1 / N} \frac{d}{d t} \int_{\left|u_{n}\right|>t} M\left(\left|\nabla u_{n}\right|\right) d x\right),
\end{aligned}
$$

then

$$
\begin{aligned}
& -\mu_{n}^{\prime}(t) \cdot M \circ H^{-1}\left(-\frac{1}{N C_{N}^{1 / N} \mu_{n}(t)^{1-1 / N}} \frac{d}{d t} \int_{\left|u_{n}\right|>t} M\left(\left|\nabla u_{n}\right|\right) d x\right) \\
& \geq N C_{N}^{1 / N} \mu_{n}(t)^{1-1 / N}\left(-\frac{1}{N C_{N}^{1 / N} \mu_{n}(t)^{1-1 / N}} \frac{d}{d t} \int_{\left|u_{n}\right|>t} M\left(\left|\nabla u_{n}\right|\right) d x\right),
\end{aligned}
$$


and also

$$
\begin{aligned}
& \frac{1}{\mu_{n}^{\prime}(t)} \frac{d}{d t} \int_{\left\{\left|u_{n}\right|>t\right\}} M\left(\left|\nabla u_{n}\right|\right) d x \\
& \quad \leq M \circ H^{-1}\left(-\frac{1}{N C_{N}^{1 / N} \mu_{n}(t)^{1-1 / N}} \frac{d}{d t} \int_{\left\{\left|u_{n}\right|>t\right\}} M\left(\left|\nabla u_{n}\right|\right) d x\right)
\end{aligned}
$$

which gives

$$
\begin{aligned}
& M^{-1}\left(\frac{1}{\mu_{n}^{\prime}(t)} \frac{d}{d t} \int_{\left\{\left|u_{n}\right|>t\right\}} M\left(\left|\nabla u_{n}\right|\right) d x\right) \\
& \quad \leq H^{-1}\left(-\frac{1}{N C_{N}^{1 / N} \mu_{n}(t)^{1-1 / N}} \frac{d}{d t} \int_{\left\{\left|u_{n}\right|>t\right\}} M\left(\left|\nabla u_{n}\right|\right) d x\right) .
\end{aligned}
$$

Let $Q \in \mathscr{A}_{M}$ and let $D(s)=M\left(Q^{-1}(s)\right), D$ is then convex, and the Jensen's inequality gives

$$
D\left(\frac{\int_{\left\{t<\left|u_{n}\right|<t+h\right\}} Q\left(\left|\nabla u_{n}\right|\right) d x}{-\mu_{n}(t+h)+\mu_{n}(t)}\right) \leq \frac{\int_{\left\{t<\left|u_{n}\right|<t+h\right\}} M\left(\left|\nabla u_{n}\right|\right) d x}{-\mu_{n}(t+h)+\mu_{n}(t)},
$$

then

$$
\begin{aligned}
& Q^{-1}\left(\frac{1}{\mu_{n}^{\prime}(t)} \frac{d}{d t} \int_{\left\{\left|u_{n}\right|>t\right\}} Q\left(\left|\nabla u_{n}\right|\right) d x\right) \\
& \quad \leq M^{-1}\left(\frac{1}{\mu_{n}^{\prime}(t)} \frac{d}{d t} \int_{\left\{\left|u_{n}\right|>t\right\}} M\left(\left|\nabla u_{n}\right|\right) d x\right) \\
& \quad \leq H^{-1}\left(-\frac{1}{N C_{N}^{1 / N} \mu_{n}(t)^{1-1 / N}} \frac{d}{d t} \int_{\left\{\left|u_{n}\right|>t\right\}} M\left(\left|\nabla u_{n}\right|\right) d x\right)
\end{aligned}
$$

which gives (3.22). By (3.21) and (3.22) and since the function

$$
t \longrightarrow \int_{\left\{\left|u_{n}\right|>t\right\}} Q\left(\left|\nabla u_{n}\right|\right) d x
$$

is absolutely continuous (see [15]), we have

$$
\begin{aligned}
\int_{\Omega} Q\left(\left|\nabla u_{n}\right|\right) d x & =\int_{0}^{\infty}\left(-\frac{d}{d t} \int_{\left\{\left|u_{n}\right|>t\right\}} Q\left(\left|\nabla u_{n}\right|\right)\right) d t \\
& \leq \int_{0}^{\infty}-\mu_{n}^{\prime}(t) Q \circ H^{-1}\left(\frac{C}{N C_{N}^{1 / N} \mu_{n}(t)^{1-1 / N}}\right) d t \\
& \leq \frac{1}{C^{\prime}} \int_{0}^{C^{\prime} \cdot \operatorname{meas}(\Omega)} Q \circ H^{-1}\left(\frac{1}{r^{1-1 / N}}\right) d r<\infty
\end{aligned}
$$


which implies that $\left(\nabla u_{n}\right)$ is bounded in $L_{Q}(\Omega)$ for each $Q \in \mathscr{A}_{M}$. Then $u_{n}$ is bounded in $W_{0}^{1} L_{Q}(\Omega)$ for each $Q \in \mathscr{A}_{M}$. Passing to a subsequence if necessary, we can assume that

$$
u_{n} \rightarrow u \quad \text { weakly in } W_{0}^{1} L_{Q}(\Omega) \text { for } \sigma\left(\prod L_{Q}, \prod E_{\bar{Q}}\right) \text {, a.e. in } \Omega \text {. }
$$

Step 3. We prove that $T_{k}\left(u_{n}\right) \rightarrow T_{k}(u)$ weakly in $W_{0}^{1} L_{M}(\Omega)$ for all $k>0$. Using the test function $T_{k}\left(u_{n}\right)$ in (3.13), we obtain

$$
\int_{\Omega} a\left(x, u_{n}, \nabla u_{n}\right) \nabla T_{k}\left(u_{n}\right) d x=\int_{\Omega} f_{n} T_{k}\left(u_{n}\right) d x+\int_{\Omega} \phi_{n}\left(u_{n}\right) \nabla T_{k}\left(u_{n}\right) d x .
$$

We claim that

$$
\int_{\Omega} \phi_{n}\left(u_{n}\right) \nabla T_{k}\left(u_{n}\right) d x=0
$$

Indeed, $\nabla T_{k}\left(u_{n}\right)=\nabla u_{n} \chi_{\left\{\left|u_{n}\right| \leq k\right\}}$, define $\theta(t)=\phi_{n}(t) \chi_{\{|t| \leq k\}}$, and $\tilde{\theta}(t)=\int_{0}^{t} \theta(\tau) d \tau$, we have by Lemma 2.2, $\tilde{\theta}\left(u_{n}\right) \in\left(W_{0}^{1} L_{M}(\Omega)\right)^{N}$,

$$
\begin{aligned}
\int_{\Omega} \phi_{n}\left(u_{n}\right) \nabla T_{k}\left(u_{n}\right) d x & =\int_{\Omega} \phi_{n}\left(u_{n}\right) \chi_{\left\{\left|u_{n}\right| \leq k\right\}} \nabla u_{n} d x \\
& =\int_{\Omega} \theta\left(u_{n}\right) \nabla u_{n} d x \\
& =\int_{\Omega} \operatorname{div}\left(\tilde{\theta}\left(u_{n}\right)\right) d x=0 \quad \text { (by Lemma 3.2) }
\end{aligned}
$$

which proves the claim.

On the other hand, (3.33) can be written as

$$
\begin{aligned}
\int_{\Omega} a\left(x, u_{n}, \nabla u_{n}\right) \nabla T_{k}\left(u_{n}\right) d x & =\int_{\Omega} a\left(x, u_{n}, \nabla T_{k}\left(u_{n}\right)\right) \nabla T_{k}\left(u_{n}\right) d x \\
& =\int_{\Omega} f_{n} T_{k}\left(u_{n}\right) d x,
\end{aligned}
$$

which implies, with (3.3), that $\nabla T_{k}\left(u_{n}\right)$ is bounded in $\left(L_{M}(\Omega)\right)^{N}$, and $T_{k}\left(u_{n}\right)$ is bounded in $\left(W_{0}^{1} L_{M}(\Omega)\right)^{N}$. Since $u_{n} \rightarrow u$ a.e. in $\Omega$ then $T_{k}\left(u_{n}\right) \rightarrow T_{k}(u)$ a.e. in $\Omega$. Then

$$
T_{k}\left(u_{n}\right) \rightarrow T_{k}(u) \quad \text { weakly in } W_{0}^{1} L_{M}(\Omega) \text { for } \sigma\left(\prod L_{M}, \prod E_{\bar{M}}\right) .
$$

Step 4. We will prove that $\nabla u_{n} \rightarrow \nabla u$ a.e. in $\Omega$. Let $\lambda>0, \epsilon>0$. For $B>1, k>0$, we consider as in [9] for $n, m \in \mathbb{N}$,

$$
\begin{aligned}
E_{1}= & \left\{\left|\nabla u_{n}\right|>B\right\} \cup\left\{\left|\nabla u_{m}\right|>B\right\} \cup\left\{\left|u_{n}\right|>B\right\} \cup\left\{\left|u_{m}\right|>B\right\}, \\
E_{2}= & \left\{\left|u_{n}-u_{m}\right|>k\right\}, \\
E_{3}= & \left\{\left|u_{n}-u_{m}\right| \leq k,\left|u_{n}\right| \leq B,\left|u_{m}\right| \leq B,\left|\nabla u_{n}\right| \leq B,\right. \\
& \left.\quad\left|\nabla u_{m}\right| \leq B,\left|\nabla u_{n}-\nabla u_{m}\right| \geq \lambda\right\},
\end{aligned}
$$

we have $\left\{\left|\nabla u_{n}-\nabla u_{m}\right| \geq \lambda\right\} \subset E_{1} \cup E_{2} \cup E_{3}$. 
Since $\left(u_{n}\right)$ and $\left(\nabla u_{n}\right)$ are bounded in $L^{1}(\Omega)$ (since $u_{n}$ is bounded in $\left.W_{0}^{1} L_{Q}(\Omega)\right)$, we have

$$
2 B \mu\left(E_{1}\right)<\int_{E_{1}}\left|\nabla u_{n}\right|+\left|u_{n}\right| d x<\int_{\Omega}\left|\nabla u_{n}\right|+\left|u_{n}\right| d x<C .
$$

Then meas $E_{1} \leq \epsilon$ for $B$ sufficiently large enough, independently of $n, m$. Thus we fix $B$ in order to have

$$
\text { meas } E_{1} \leq \epsilon .
$$

Now we claim that meas $E_{3} \leq \epsilon$ for $n$ and $m$ large. Let $C_{1}$ be such that $\left\|u_{n}\right\|_{1} \leq$ $C_{1}$ and $\left\|\nabla u_{n}\right\|_{1} \leq C_{1}$. As in [9], the assumption (3.2) gives the existence of a measurable function $\gamma(x)$ such that

$$
\begin{gathered}
\operatorname{meas}(\{x \in \Omega: \gamma(x)=0\})=0, \\
{[a(x, t, \xi)-a(x, t, \bar{\xi})][\xi-\bar{\xi}] \geq \gamma(x)>0,}
\end{gathered}
$$

for all $t \in \mathbb{R}, \xi, \bar{\xi} \in \mathbb{R}^{N},|t|,|\xi|,|\bar{\xi}| \leq B,|\xi-\bar{\xi}| \geq \lambda$ a.e. in $\Omega$. We have

$$
\begin{aligned}
\int_{E_{3}} \gamma(x) d x \leq & \int_{E_{3}}\left[a\left(x, u_{n}, \nabla u_{n}\right)-a\left(x, u_{n}, \nabla u_{m}\right)\right]\left[\nabla u_{n}-\nabla u_{m}\right] d x \\
\leq & \int_{E_{3}}\left[a\left(x, u_{m}, \nabla u_{m}\right)-a\left(x, u_{n}, \nabla u_{m}\right)\right]\left[\nabla u_{n}-\nabla u_{m}\right] d x \\
& +\int_{E_{3}}\left[a\left(x, u_{n}, \nabla u_{n}\right)-a\left(x, u_{m}, \nabla u_{m}\right)\right]\left[\nabla u_{n}-\nabla u_{m}\right] d x
\end{aligned}
$$

Using the test function $T_{k}\left(u_{n}-u_{m}\right)$ in (3.13) and integrating on $E_{3}$, we obtain

$$
\begin{aligned}
\int_{E_{3}}\left[a\left(x, u_{n}, \nabla u_{n}\right)-a\left(x, u_{m}, \nabla u_{m}\right)\right] \nabla T_{k}\left(u_{n}-u_{m}\right) d x \\
=\int_{E_{3}}\left(f_{n}-f_{m}\right) T_{k}\left(u_{n}-u_{m}\right) d x \\
\quad+\int_{E_{3}}\left[\phi_{n}\left(u_{n}\right)-\phi_{m}\left(u_{m}\right)\right] \nabla T_{k}\left(u_{n}-u_{m}\right) d x,
\end{aligned}
$$

with

$$
\begin{gathered}
\int_{E_{3}}\left[\phi_{n}\left(u_{n}\right)-\phi_{m}\left(u_{m}\right)\right] \nabla T_{k}\left(u_{n}-u_{m}\right) d x \\
\leq 2 B \int_{E_{3}}\left|\phi_{n}\left(u_{n}\right)-\phi_{m}\left(u_{m}\right)\right| d x \\
\leq 2 B \int_{E_{3}}\left[\left|\phi\left(T_{n}\left(u_{n}\right)\right)-\phi\left(u_{n}\right)\right|+\left|\phi\left(u_{n}\right)-\phi\left(u_{m}\right)\right|\right. \\
\left.+\left|\phi\left(u_{m}\right)-\phi\left(T_{m}\left(u_{m}\right)\right)\right|\right] d x .
\end{gathered}
$$


Let $n_{0} \geq B$, then for $n, m \geq n_{0}$ we have $T_{n}\left(u_{n}\right)=u_{n}$ and $T_{m}\left(u_{m}\right)=u_{m}$ on $E_{3}$, which implies that the first and the third integral of the last inequality vanish. The second integral of (3.42) is bounded for $n, m \geq n_{0}$ by

$$
2 k\|f\|_{1, \Omega}+2 B \int_{E_{3}}\left|\phi\left(u_{n}\right)-\phi\left(u_{m}\right)\right| d x .
$$

For a.e. $x \in \Omega$ and $\epsilon_{1}>0$ there exist $\eta(x) \geq 0(\operatorname{meas}\{x \in \Omega: \eta(x)=0\}=0)$ such that $\left|s-s^{\prime}\right| \leq \eta(x),|s|,\left|s^{\prime}\right|,|\xi| \leq B$ implies

$$
\left|a(x, s, \xi)-a\left(x, s^{\prime}, \xi\right)\right| \leq \epsilon_{1} .
$$

We use now the continuity of $\phi$, to obtain for a.e. $x \in \Omega$ and $\epsilon_{2}>0, \eta_{1}(x) \geq$ $0\left(\operatorname{meas}\left\{x \in \Omega: \eta_{1}(x)=0\right\}=0\right)$ such that $\left|s-s^{\prime}\right| \leq \eta_{1}(x),|s|,\left|s^{\prime}\right| \leq B$ implies

$$
\left|\phi(s)-\phi\left(s^{\prime}\right)\right| \leq \epsilon_{2}
$$

Then

$$
\begin{aligned}
\int_{E_{3}} \gamma(x) d x \leq \int_{E_{3} \cap\{x \in \Omega: \eta(x)<k\}}\left[a\left(x, u_{m}, \nabla u_{m}\right)-a\left(x, u_{n}, \nabla u_{m}\right)\right] \\
\times\left[\nabla u_{n}-\nabla u_{m}\right] d x \\
+\int_{E_{3} \cap\{x \in \Omega: \eta(x) \geq k\}}\left[a\left(x, u_{m}, \nabla u_{m}\right)-a\left(x, u_{n}, \nabla u_{m}\right)\right] \\
\times\left[\nabla u_{n}-\nabla u_{m}\right] d x \\
+2 k\|f\|_{1, \Omega}+2 B \int_{E_{3} \cap\left\{x \in \Omega: \eta_{1}(x)<k\right\}}\left|\phi\left(u_{n}\right)-\phi\left(u_{m}\right)\right| d x \\
+\int_{E_{3} \cap\left\{x \in \Omega: \eta_{1}(x) \geq k\right\}}\left|\phi\left(u_{n}\right)-\phi\left(u_{m}\right)\right| d x .
\end{aligned}
$$

By using for the first integral the definition of $E_{3}$ and condition (3.1), for the second integral the definition of $E_{3}$ and (3.46), for the fourth integral the definition of $E_{3}$ and $\left|\phi\left(u_{n}\right)\right| \leq C(B)$ (since $\left|u_{n}\right| \leq B$ and $\phi$ continuous), and for the last integral the definition of $E_{3}$ and (3.47), we obtain

$$
\begin{aligned}
\int_{E_{3}} \gamma(x) d x \leq & C^{\prime}(B) \int_{E_{3} \cap\{x \in \Omega: \eta(x)<k\}}[1+d(x)] d x+2 C_{1}(B) \epsilon_{1} \\
& +2 k\|f\|_{1, \Omega}+2 C(B) \text { meas }\left\{x \in \Omega: \eta_{1}(x)<k\right\}+C_{2} \epsilon_{2} .
\end{aligned}
$$

We have meas $\{x \in \Omega: \eta(x)<k\} \rightarrow 0$ when $k \rightarrow 0$, and meas $\left\{x \in \Omega: \eta_{1}(x)<\right.$ $k\} \rightarrow 0$ when $k \rightarrow 0$. Let $\epsilon>0$ and let $\delta$ be the real, in Lemma 3.4, corresponding to $\epsilon$, we choose $\epsilon_{1}, \epsilon_{2}$ such that

$$
2 C_{1}(B) \epsilon_{1} \leq \frac{\delta}{5}, \quad C_{2} \epsilon_{2} \leq \frac{\delta}{5}
$$


and $k$ such that

$$
\begin{gathered}
C^{\prime}(B) \int_{E_{3} \cap\{x \in \Omega: \eta(x)<k\}}[1+d(x)] d x<\frac{\delta}{5}, \quad 2 k\|f\|_{1, \Omega} \leq \frac{\delta}{5}, \\
2 C(B) \text { meas }\left\{x \in \Omega: \eta_{1}(x)<k\right\}<\frac{\delta}{5} .
\end{gathered}
$$

Then $\int_{E_{3}} \gamma(x) d x<\delta$ and Lemma 3.4 implies that

$$
\text { meas } E_{3}<\epsilon \quad \forall n, m \geq n_{0} .
$$

This completes the proof of the claim.

Let the last $k$ be fixed, $u_{n}$ a Cauchy sequence in measure, we choose $n_{1}$ such that

$$
\text { meas } E_{2} \leq \epsilon \quad \forall n, m \geq n_{1} .
$$

Then

$$
\text { meas }\left\{x \in \Omega:\left|\nabla u_{n}-\nabla u_{m}\right| \geq \lambda\right\} \leq \epsilon \quad \forall n, m \geq \max \left(n_{1}, n_{0}\right)
$$

and $\nabla u_{n} \rightarrow \nabla u$ in measure, consequently

$$
\nabla u_{n} \longrightarrow \nabla u \quad \text { a.e. in } \Omega \text {. }
$$

Step 5. Let $\varphi \in W_{0}^{1} L_{M}(\Omega) \cap L^{\infty}(\Omega)$. From Lemma 2.3, there exists a sequence $\left(\varphi_{j}\right) \in \mathscr{D}(\Omega)$ such that $\varphi_{j}$ converges to $\varphi$ for the modular convergence in $W_{0}^{1} L_{M}(\Omega)$ with

$$
\left\|\varphi_{j}\right\|_{L^{\infty}(\Omega)} \leq(N+1)\|\varphi\|_{L^{\infty}(\Omega)} .
$$

Using $T_{k}\left[u_{n}-\varphi_{j}\right]$ as a test function in (3.13) we obtain

$$
\begin{aligned}
& \int_{\Omega} a\left(x, u_{n}, \nabla u_{n}\right) \nabla T_{k}\left[u_{n}-\varphi_{j}\right] d x \\
& \quad=\int_{\Omega} f_{n} T_{k}\left[u_{n}-\varphi_{j}\right] d x+\int_{\Omega} \phi_{n}\left(u_{n}\right) \nabla T_{k}\left[u_{n}-\varphi_{j}\right] d x
\end{aligned}
$$

which gives, if $n \rightarrow \infty$,

$$
\begin{aligned}
& \liminf _{n \rightarrow \infty} \int_{\Omega} a\left(x, u_{n}, \nabla u_{n}\right) \nabla T_{k}\left[u_{n}-\varphi_{j}\right] d x \\
& \geq \liminf _{n \rightarrow \infty} \int_{\Omega}\left[a\left(x, u_{n}, \nabla u_{n}\right)-a\left(x, u_{n}, \nabla \varphi_{j}\right)\right] \nabla T_{k}\left[u_{n}-\varphi_{j}\right] d x \\
& \quad+\lim _{n \rightarrow \infty} \int_{\Omega} a\left(x, T_{k+\left\|\varphi_{j}\right\|_{L(\Omega)}}\left(u_{n}\right), \nabla \varphi_{j}\right) \nabla T_{k}\left[u_{n}-\varphi_{j}\right] d x \\
& \geq \int_{\Omega}\left[a(x, u, \nabla u)-a\left(x, u, \nabla \varphi_{j}\right)\right] \nabla T_{k}\left[u-\varphi_{j}\right] d x \\
& \quad+\int_{\Omega} a\left(x, u, \nabla \varphi_{j}\right) \nabla T_{k}\left[u-\varphi_{j}\right] d x,
\end{aligned}
$$


where we have used Fatou lemma for the first integral, and for the second the convergences $\nabla T_{k}\left[u_{n}-\varphi_{j}\right] \rightarrow \nabla T_{k}\left[u-\varphi_{j}\right]$ by (3.37) in $\left(L_{M}(\Omega)\right)^{N}$ for $\sigma\left(\prod L_{M}\right.$, $\left.\prod E_{\bar{M}}\right)$ and $a\left(x, T_{k+\left\|\varphi_{j}\right\|_{L^{\infty}(\Omega)}}\left(u_{n}\right), \nabla \varphi_{j}\right) \rightarrow a\left(x, T_{k+\left\|\varphi_{j}\right\|_{L^{\infty}(\Omega)}}(u), \nabla \varphi_{j}\right)$ strongly in $\left(E_{\bar{M}}(\Omega)\right)^{N}$ by $(3.1)$, which implies that

$$
\liminf _{n \rightarrow \infty} \int_{\Omega} a\left(x, u_{n}, \nabla u_{n}\right) \nabla T_{k}\left[u_{n}-\varphi_{j}\right] d x \geq \int_{\Omega} a(x, u, \nabla u) \nabla T_{k}\left[u-\varphi_{j}\right] d x .
$$

For $n \geq k+(N+1)\|\varphi\|_{L^{\infty}(\Omega)}$,

$$
\begin{aligned}
\int_{\Omega} \phi_{n}\left(u_{n}\right) \nabla T_{k}\left[u_{n}-\varphi_{j}\right] d x & =\int_{\Omega} \phi\left(T_{k+(N+1)\|\varphi\|_{L^{\infty}(\Omega)}}\left(u_{n}\right)\right) \nabla T_{k}\left[u_{n}-\varphi_{j}\right] d x \\
& \underset{n \rightarrow \infty}{\longrightarrow} \int_{\Omega} \phi\left(T_{k+(N+1)\|\varphi\|_{L^{\infty}(\Omega)}}(u)\right) \nabla T_{k}\left[u-\varphi_{j}\right] d x,
\end{aligned}
$$

we have used the convergences $\nabla T_{k}\left[u_{n}-\varphi_{j}\right] \rightarrow \nabla T_{k}\left[u-\varphi_{j}\right]$ by $(3.37)$ in $\left(L_{M}(\Omega)\right)^{N}$ and $\phi\left(T_{k+(N+1)\|\varphi\|_{L^{\infty}(\Omega)}}\left(u_{n}\right)\right) \rightarrow \phi\left(T_{k+(N+1)\|\varphi\|_{L^{\infty}(\Omega)}}(u)\right)$ strongly in $\left(E_{\bar{M}}(\Omega)\right)^{N}$ since $\phi$ is continuous. On the other hand, since $f_{n} \rightarrow f$ strongly in $L^{1}(\Omega)$ and $T_{k}\left[u_{n}-\right.$ $\left.\varphi_{j}\right] \rightarrow T_{k}\left[u-\varphi_{j}\right]$ weakly* in $L^{\infty}(\Omega)$, we have

$$
\int_{\Omega} f_{n} T_{k}\left[u_{n}-\varphi_{j}\right] d x \longrightarrow \int_{\Omega} f T_{k}\left[u-\varphi_{j}\right] d x
$$

Then

$$
\begin{aligned}
\int_{\Omega} a(x, u, \nabla u) \nabla T_{k}\left[u-\varphi_{j}\right] d x \geq & \int_{\Omega} \phi\left(T_{k+(N+1)\|\varphi\|_{L^{\infty}(\Omega)}}(u)\right) \nabla T_{k}\left[u-\varphi_{j}\right] d x \\
& +\int_{\Omega} f T_{k}\left[u-\varphi_{j}\right] d x .
\end{aligned}
$$

Now, if $j \rightarrow \infty$ in (3.62), we get

$$
\begin{aligned}
& \liminf _{j \rightarrow \infty} \int_{\Omega} a(x, u, \nabla u) \nabla T_{k}\left[u-\varphi_{j}\right] d x \\
& \geq \liminf _{j \rightarrow \infty} \int_{\Omega}\left[a(x, u, \nabla u)-a\left(x, u, \nabla \varphi_{j}\right)\right] \nabla T_{k}\left[u-\varphi_{j}\right] d x \\
& \quad+\lim _{j \rightarrow \infty} \int_{\Omega} a\left(x, u, \nabla \varphi_{j}\right) \nabla T_{k}\left[u-\varphi_{j}\right] d x \\
& \geq \int_{\Omega}[a(x, u, \nabla u)-a(x, u, \nabla \varphi)] \nabla T_{k}[u-\varphi] d x \\
& \quad+\int_{\Omega} a(x, u, \nabla \varphi) \nabla T_{k}[u-\varphi] d x,
\end{aligned}
$$

where we have used Fatou lemma for the first integral, and for the second the convergences $\nabla T_{k}\left[u-\varphi_{j}\right] \rightarrow \nabla T_{k}[u-\varphi]$ in $\left(L_{M}(\Omega)\right)^{N}$ for the modular convergence and $a\left(x, u, \nabla \varphi_{j}\right) \rightarrow a(x, u, \nabla \varphi)$ in $\left(L_{\bar{M}}(\Omega)\right)^{N}$ for the modular convergence, 
which implies that

$$
\liminf _{j \rightarrow \infty} \int_{\Omega} a(x, u, \nabla u) \nabla T_{k}\left[u-\varphi_{j}\right] d x \geq \int_{\Omega} a(x, u, \nabla u) \nabla T_{k}[u-\varphi] d x
$$

On the other hand, since $\nabla T_{k}\left[u-\varphi_{j}\right] \rightarrow \nabla T_{k}[u-\varphi]$ in $\left(L_{M}(\Omega)\right)^{N}$ for the modular convergence, then weakly for $\sigma\left(\prod L_{M}, \prod L_{\bar{M}}\right)$ and $\phi\left(T_{k+(N+1)\|\varphi\|_{L^{\infty}(\Omega)}}(u)\right) \in$ $\left(L_{\bar{M}}(\Omega)\right)^{N}$ we have

$$
\begin{aligned}
\int_{\Omega} \phi & \left(T_{k+(N+1)\|\varphi\|_{L^{\infty}(\Omega)}}(u)\right) \nabla T_{k}\left[u-\varphi_{j}\right] d x \\
& \underset{j \rightarrow \infty}{\longrightarrow} \int_{\Omega} \phi\left(T_{k+(N+1)\|\varphi\|_{L^{\infty}(\Omega)}}(u)\right) \nabla T_{k}[u-\varphi] d x \\
& =\int_{\Omega} \phi(u) \nabla T_{k}[u-\varphi] d x .
\end{aligned}
$$

Since $f \in L^{1}(\Omega)$ and $T_{k}\left[u-\varphi_{j}\right] \rightarrow T_{k}[u-\varphi]$ weakly* in $L^{\infty}(\Omega)$, we have

$$
\int_{\Omega} f T_{k}\left[u-\varphi_{j}\right] d x \longrightarrow \int_{\Omega} f T_{k}[u-\varphi] d x
$$

Then

$$
\int_{\Omega} a(x, u, \nabla u) \nabla T_{k}[u-\varphi] d x \geq \int_{\Omega} \phi(u) \nabla T_{k}[u-\varphi] d x+\int_{\Omega} f T_{k}[u-\varphi] d x
$$

and $u$ is an entropy solution of problem (1.1).

Theorem 3.7. Suppose, in Theorem 3.5, that the $N$-function $M$ satisfies, furthermore, the $\Delta_{2}$-condition and $f \geq 0$, then the entropy solution $u$ of problem (1.1) satisfies $u \geq 0$.

Proof of Theorem 3.7. Using $\varphi=T_{l}\left(u^{+}\right)$as test function in the definition of entropy solution, we obtain

$$
\begin{aligned}
& \int_{\Omega} a(x, u, \nabla u) \nabla T_{k}\left[u-T_{l}\left(u^{+}\right)\right] d x \\
& \quad \leq \int_{\Omega} f T_{k}\left[u-T_{l}\left(u^{+}\right)\right] d x+\int_{\Omega} \phi(u) \nabla T_{k}\left[u-T_{l}\left(u^{+}\right)\right] d x .
\end{aligned}
$$

We have

$$
\int_{\Omega} f T_{k}\left[u-T_{l}\left(u^{+}\right)\right] d x \leq \int_{\{u \geq l\}} f T_{k}\left[u-T_{l}(u)\right] d x
$$


Indeed,

$$
\begin{aligned}
\int_{\Omega} f T_{k}\left[u-T_{l}\left(u^{+}\right)\right] d x= & \int_{u \geq l} f T_{k}\left[u-T_{l}\left(u^{+}\right)\right] d x \\
& +\int_{0<u<l} f T_{k}\left[u-T_{l}\left(u^{+}\right)\right] d x \\
& +\int_{u \leq 0} f T_{k}\left[u-T_{l}\left(u^{+}\right)\right] d x
\end{aligned}
$$

If $0<u<l$ then $u-T_{l}\left(u^{+}\right)=0$ and $\int_{0<u<l} f T_{k}\left[u-T_{l}\left(u^{+}\right)\right] d x=0$. If $u \leq 0$ then $u-T_{l}\left(u^{+}\right)=u$ and $\int_{u \leq 0} f T_{k}\left[u-T_{l}\left(u^{+}\right)\right] d x \leq 0$ since $f$ is positive. If $u \geq l$ then $u^{+}=u$ and $\int_{u \geq l} f T_{k}\left[u-T_{l}\left(u^{+}\right)\right] d x \leq \int_{u \geq l} f T_{k}\left[u-T_{l}(u)\right] d x$.

On the other hand, we claim that

$$
\int_{\Omega} \phi(u) \nabla T_{k}\left[u-T_{l}\left(u^{+}\right)\right] d x=0 .
$$

Indeed, if $0<u<l$, then $u-T_{l}\left(u^{+}\right)=0, \int_{0<u<l} \phi(u) \nabla T_{k}\left[u-T_{l}\left(u^{+}\right)\right] d x=0$. If $u \leq 0$, then $u-T_{l}\left(u^{+}\right)=u$,

$$
\begin{aligned}
\int_{u \leq 0} \phi(u) \nabla T_{k}\left[u-T_{l}\left(u^{+}\right)\right] d x & =\int_{-k \leq u \leq 0} \phi(u) \nabla u d x \\
& =\int_{\Omega} \phi(u) \nabla u \chi_{\{-k \leq u \leq 0\}} d x .
\end{aligned}
$$

We verify that the third integral of the last inequality vanishes. For this, define $\theta(t)=\phi(t) \chi_{\{-k \leq t \leq 0\}}$, and $\tilde{\theta}(t)=\int_{0}^{t} \theta(\tau) d \tau$ we have, by Lemma 2.2, $\tilde{\theta}(u) \in$ $\left(W_{0}^{1} L_{M}(\Omega)\right)^{N}$ which implies

$$
\begin{aligned}
\int_{\Omega} \phi(u) \nabla u \chi_{\{-k \leq u \leq 0\}} d x & =\int_{\Omega} \theta(u) \nabla u d x \\
& =\int_{\Omega} \operatorname{div}(\tilde{\theta}(u)) d x=0 \quad \text { (by Lemma 3.2). }
\end{aligned}
$$

If $u \geq l$ then $u^{+}=u$ and

$$
\begin{aligned}
\int_{\{u \geq l\}} \phi(u) \nabla T_{k}\left[u-T_{l}\left(u^{+}\right)\right] d x & =\int_{l \leq u \leq l+k} \phi(u) \nabla u d x \\
& =\int_{\Omega} \phi(u) \nabla u \chi_{\{l \leq u \leq l+k\}} d x .
\end{aligned}
$$

Similarly, we verify that

$$
\int_{\Omega} \phi(u) \nabla u \chi_{\{l \leq u \leq l+k\}} d x=0 .
$$


This completes the proof of the claim which implies that

$$
\int_{\Omega} a(x, u, \nabla u) \nabla T_{k}\left[u-T_{l}\left(u^{+}\right)\right] d x \leq \int_{u \geq l} f T_{k}\left[u-T_{l}(u)\right] d x
$$

or

$$
\begin{aligned}
& \int_{\Omega} a(x, u, \nabla u) \nabla T_{k}\left[u-T_{l}\left(u^{+}\right)\right] d x \\
& \quad=\int_{l \leq u \leq l+k} a(x, u, \nabla u) \nabla u d x+\int_{-k \leq u \leq 0} a(x, u, \nabla u) \nabla u d x \\
& \quad \geq \int_{l \leq u \leq l+k} M\left(\frac{|\nabla u|}{\lambda}\right) d x+\int_{-k \leq u \leq 0} M\left(\frac{|\nabla u|}{\lambda}\right) d x,
\end{aligned}
$$

which gives

$$
\int_{l \leq u \leq l+k} M\left(\frac{|\nabla u|}{\lambda}\right) d x+\int_{-k \leq u \leq 0} M\left(\frac{|\nabla u|}{\lambda}\right) d x \leq \int_{u \geq l} f T_{k}\left[u-T_{l}(u)\right] d x
$$

Letting $l \rightarrow \infty$ in (3.78) we have

$$
\begin{aligned}
\int_{u \geq l} f T_{k}\left[u-T_{l}(u)\right] d x & \longrightarrow \quad \text { since } f T_{k}[2 u] \in L^{1}(\Omega), \\
\int_{l \leq u \leq l+k} M\left(\frac{|\nabla u|}{\lambda}\right) d x & \geq \int_{l \leq u \leq k} M\left(\frac{|\nabla u|}{\lambda}\right) d x \\
& =\int_{l \leq u} M\left(\frac{\left|\nabla T_{k}(u)\right|}{\lambda}\right) d x \\
& \longrightarrow 0, \quad \text { when } l \longrightarrow \infty
\end{aligned}
$$

since $M\left(\left|\nabla T_{k}(u)\right| / \lambda\right) \in L^{1}(\Omega)$ and $M$ satisfies the $\Delta_{2}$-condition. Then

$$
\int_{-k \leq u \leq 0} M\left(\frac{|\nabla u|}{\lambda}\right) d x=0 \quad \forall k
$$

which implies that,

$$
\begin{gathered}
\int_{u \leq 0} M\left(\frac{|\nabla u|}{\lambda}\right) d x=\int_{\Omega} M\left(\frac{\left|\nabla u^{-}\right|}{\lambda}\right) d x=0 \\
\nabla u^{-}=0, \quad u^{-}=c \quad \text { a.e. in } \Omega .
\end{gathered}
$$

Or $u^{-} \in W_{0}^{1} L_{Q}(\Omega)$ then $u^{-}=0$ a.e. in $\Omega$ which proves that

$$
u \geq 0 \quad \text { a.e. in } \Omega \text {. }
$$




\section{References}

[1] R. A. Adams, Sobolev Spaces, Pure and Applied Mathematics, vol. 65, Academic Press, New York, 1975.

[2] M. K. Alaoui, Sur certains problèmes elliptiques avec second membre mesure ou $L_{\text {loc }}^{1}$, Ph.D. thesis, Université Sidi Mohammed Ben Abdallah, Morocco, 1999.

[3] P. Bénilan, L. Boccardo, T. Gallouët, R. Gariepy, M. Pierre, and J. L. Vázquez, An L $L^{1}-$ theory of existence and uniqueness of solutions of nonlinear elliptic equations, Ann. Scuola Norm. Sup. Pisa Cl. Sci. (4) 22 (1995), no. 2, 241-273.

[4] A. Benkirane and J. Bennouna, Existence of renormalized solutions for some elliptic problems involving derivatives of nonlinear terms in Orlicz spaces, to appear in Lecture Notes in Pure and Applied Mathematics, Marcel Dekker.

[5] A. Benkirane and A. Elmahi, Almost everywhere convergence of the gradients of solutions to elliptic equations in Orlicz spaces and application, Nonlinear Anal. 28 (1997), no. 11, 1769-1784.

[6] An existence theorem for a strongly nonlinear elliptic problem in Orlicz spaces, Nonlinear Anal. 36 (1999), no. 1, 11-24.

[7] A. Benkirane and J.-P. Gossez, An approximation theorem in higher order OrliczSobolev spaces and applications, Studia Math. 92 (1989), no. 3, 231-255.

[8] L. Boccardo, Some nonlinear Dirichlet problems in $L^{1}$ involving lower order terms in divergence form, Progress in Elliptic and Parabolic Partial Differential Equations (Capri, 1994), Pitman Res. Notes Math. Ser., vol. 350, Longman, Harlow, 1996, pp. 43-57.

[9] L. Boccardo and T. Gallouët, Nonlinear elliptic equations with right-hand side measures, Comm. Partial Differential Equations 17 (1992), no. 3-4, 641-655.

[10] R. J. DiPerna and P.-L. Lions, On the Cauchy problem for Boltzmann equations: global existence and weak stability, Ann. of Math. (2) 130 (1989), no. 2, 321-366.

[11] J.-P. Gossez, Some approximation properties in Orlicz-Sobolev spaces, Studia Math. 74 (1982), no. 1, 17-24.

[12] J.-P. Gossez and V. Mustonen, Variational inequalities in Orlicz-Sobolev spaces, Nonlinear Anal. 11 (1987), no. 3, 379-392.

[13] A. M. Krasnosel'skii and Ya Rutickii, Convex Functions and Orlicz Spaces, Noodhoff, Groningen, The Netherlands, 1969.

[14] P. L. Lions and F. Murat, Sur les solutions d'equations elliptiques non linéaires, to appear in C. R. Acad. Sci. Paris.

[15] G. Talenti, Nonlinear elliptic equations, rearrangements of functions and Orlicz spaces, Ann. Mat. Pura Appl. (4) 120 (1979), 160-184.

A. Benkirane and J. Bennouna: Département de Mathématique, Faculté des Sciences Dhar Mehraz, Université Sidi Mohammed Ben Abdallah, BP 1796 Atlas Fes, Morocco 


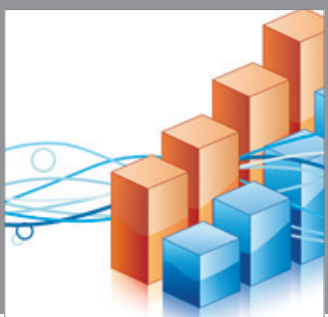

Advances in

Operations Research

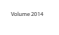

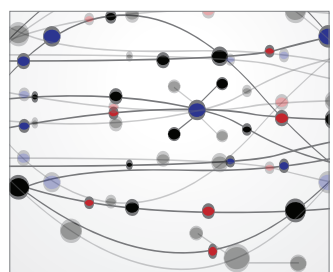

\section{The Scientific} World Journal
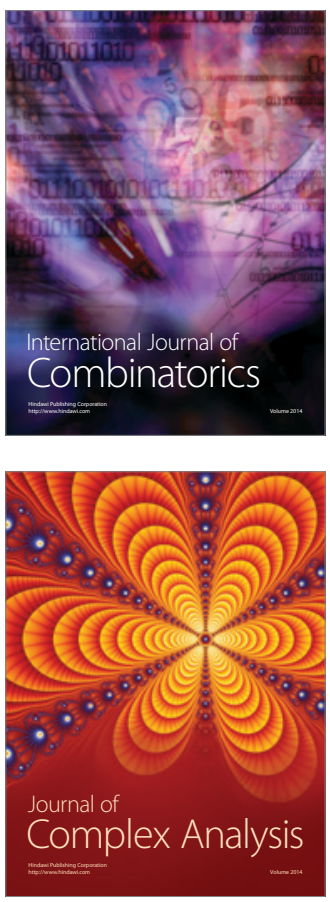

International Journal of

Mathematics and

Mathematical

Sciences
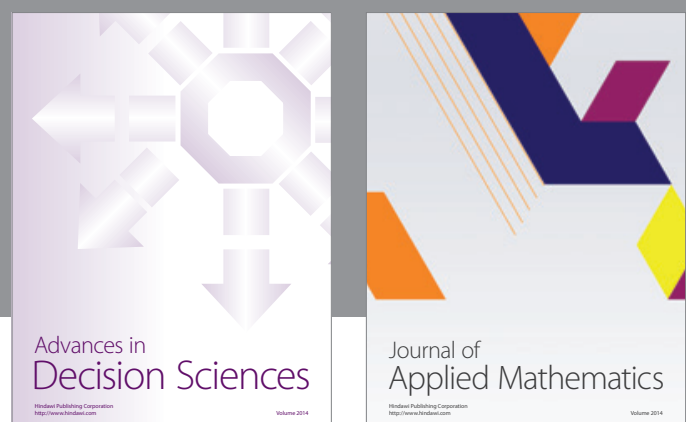

Journal of

Applied Mathematics
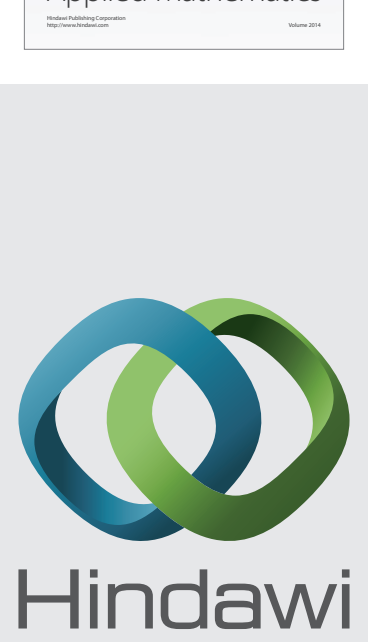

Submit your manuscripts at http://www.hindawi.com
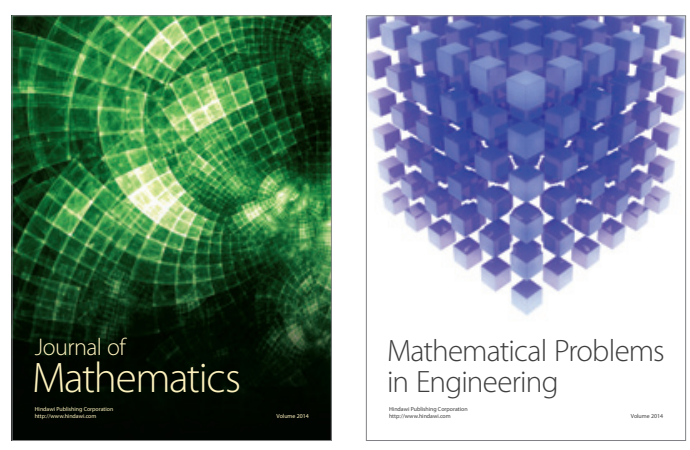

Mathematical Problems in Engineering
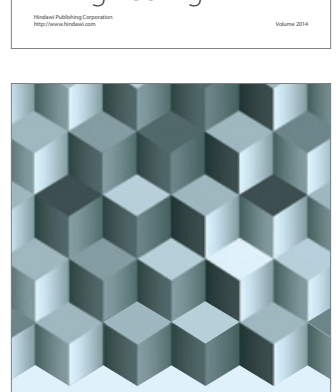

Journal of

Function Spaces
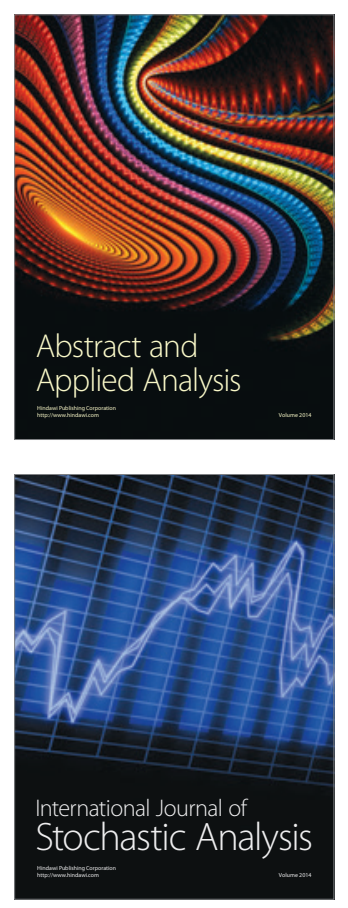

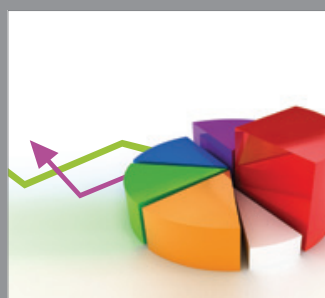

ournal of

Probability and Statistics

Promensencen
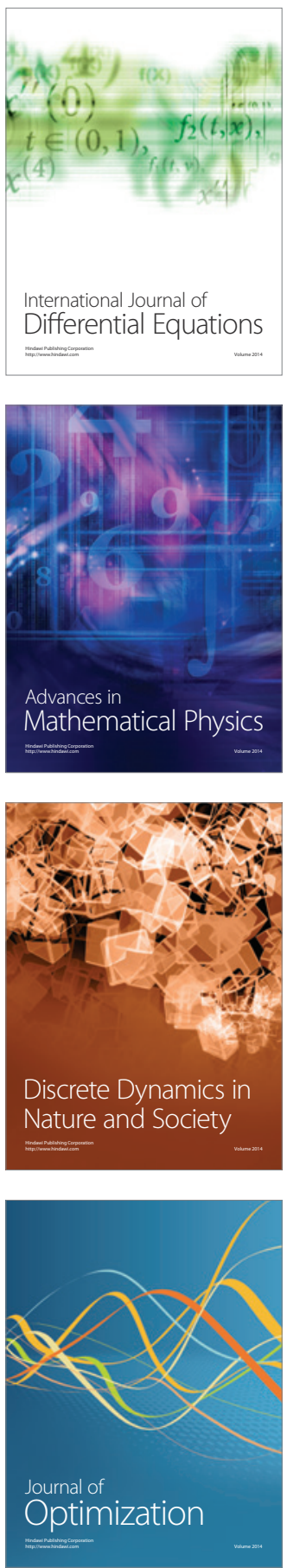